\title{
Asymptotic upper bounds for the entropy of orthogonal polynomials in the Szegö class
}

\author{
B. Beckermann \\ Department of Mathematics, Universite de Lille I, France \\ A. Martínez-Finkelshtein* \\ Department of Statistics and Applied Mathematics, University of Almería \\ and Institute Carlos I for Theoretical and Computational Physics, \\ Granada University, Spain \\ E.A. Rakhmanov \\ Department of Mathematics, University of South Florida, USA \\ F. Wielonsky \\ Department of Mathematics, Universite de Lille I, \\ and INRIA Sophia Antipolis, France
}

\begin{abstract}
We give an asymptotic upper bound as $n \rightarrow \infty$ for the entropy integral$$
E_{n}(w)=-\int p_{n}^{2}(x) \log \left(p_{n}^{2}(x)\right) w(x) d x,
$$

where $p_{n}$ is the $n$th degree orthonormal polynomial with respect to a weight $w(x)$ on $[-1,1]$ which belongs to the Szegő class. We also study two functionals closely related to the entropy integral. First, their asymptotic behavior is completely described for weights $w$ in the Bernstein class. Then, as for the entropy, we obtain asymptotic upper bounds for these two functionals when $w(x)$ belongs to the Szegö class. In each case, we give conditions for these upper bounds to be attained.
\end{abstract}

\section{Introduction}

In the framework of the density functional theory (see e.g. [7, 12]) the physical and chemical properties of fermionic systems are described by means of the single-particle probability densities. If $\Psi(\vec{r})$ is the wave function of a single-particle system in a (D-dimensional) position space, and $\widehat{\Psi}(\vec{p})$ is the corresponding wave function in momentum space (that is, the Fourier transform of $\Psi(\vec{r})$ ), then the position and momentum densities of the system are given by

$$
\rho(\vec{r})=|\Psi(\vec{r})|^{2}, \quad \gamma(\vec{p})=|\widehat{\Psi}(\vec{p})|^{2},
$$

${ }^{*}$ Corresponding author. E-mail: andrei@ual.es 
respectively. It is known that the Boltzmann-Gibbs-Shannon position-space entropy

$$
S(\rho)=-\int \rho(\vec{r}) \ln \rho(\vec{r}) d \vec{r}
$$

measures the uncertainty in the localization of the particle in space (lower entropy indicates a more concentrated wave function, with the associated higher accuracy in predicting the localization of the particle). Similar is true for the momentum-space entropy

$$
S(\gamma)=-\int \gamma(\vec{p}) \ln \gamma(\vec{p}) d \vec{p} .
$$

These quantities have importance in the study of the structure and dynamics of atomic and molecular systems; we refer the reader to the survey [5] and to references therein. Both $S(\rho)$ and $S(\gamma)$ also play role in a generalization of the Heisenberg uncertainty relation: it has been established [3] that for any pair of densities $\rho(\vec{r})$ and $\gamma(\vec{p})$ in $D$-dimensional space, we have the sharp inequality

$$
S(\rho)+S(\gamma) \geq D(1+\ln \pi),
$$

which expresses quantitatively the impossibility of simultaneous localization of a pair of observables with no common eigenstates.

It is well known that the wave function of many important systems, such as $D$-dimensional harmonic oscillator and hydrogen atom, are expressible in terms of families of orthogonal polynomials. It is not surprising that, as it has been shown in [6, 15, the computation of the entropies $S(\rho)$ and $S(\gamma)$ usually can be reduced to integrals involving these polynomials.

Let $\nu$ be a positive unit Borel measure on $\Delta:=[-1,1]$ and let

$$
p_{n}(x)=\gamma_{n} \prod_{j=1}^{n}\left(x-\zeta_{j}^{(n)}\right), \quad \gamma_{n}>0, \quad n \in \mathbb{N},
$$

denote the corresponding sequence of orthonormal polynomials such that

$$
\int p_{n}(x) p_{m}(x) d \nu(x)=\delta_{m n}, \quad m, n \in \mathbb{N} .
$$

We define the information entropy of the polynomials $p_{n}(x)$ as

$$
E_{n}=E_{n}(\nu)=-\int p_{n}^{2}(x) \ln \left(p_{n}^{2}(x)\right) d \nu(x) .
$$

Throughout the paper, we will assume that the orthogonality measure $\nu$ is absolutely continuous with respect to the Lebesgue measure $\lambda$ on $\Delta$ with Radon-Nikodym derivative

$$
d \nu / d \lambda=\nu^{\prime}(x)=w(x), \quad w \in L^{1}(\Delta) .
$$

For normalization purposes, we will always assume that the weight $w$ is unitary, i.e.

$$
\int_{\Delta} w(x) d x=1
$$


The information entropy will be indistinctly denoted by $E_{n}(\nu)$ and $E_{n}(w)$. We follow this convention below for other notations.

The asymptotic behavior of $E_{n}$ as $n \rightarrow \infty$ has a special interest in the study of the so-called Rydberg states of quantum-mechanical systems. Besides physical motivations, there are some fascinating aspects of this problem because of a certain universal behavior of related integrals, and because of a close connection of the entropy $E_{n}$ with important functionals of the normalized zero counting measures of the polynomials $p_{n}$,

$$
\mu_{n}=\frac{1}{n} \sum_{j=1}^{n} \delta_{\zeta_{j}^{(n)}}, \quad n>0,
$$

and of the following probability measures $\nu_{n}$ :

$$
d \nu_{n}(x)=p_{n}^{2}(x) d \nu(x), \quad n \geq 0,
$$

(note that $\nu_{0}=\nu$ ). Both measures are standard objects of study in the analytic theory of orthogonal polynomials. For instance, the normalized zero counting measure $\mu_{n}$ is closely connected with the $n$-th root asymptotics of $p_{n}$, and as was shown in [13, $\nu_{n}$ is associated with the behavior of the ratio $p_{n+1} / p_{n}$ as $n \rightarrow \infty$.

If $\mu$ and $\nu$ are positive Borel measures on $\mathbb{C}$, then their mutual entropy is defined as

$$
S(\mu, \nu)= \begin{cases}-\infty, & \text { if } \mu \text { is not } \nu \text {-absolutely continuous, } \\ -\int \log \left(\frac{d \mu}{d \nu}\right) d \mu, & \text { if } \mu \text { is } \nu \text {-absolutely continuous, }\end{cases}
$$

and their mutual logarithmic energy as

$$
I(\nu, \mu)=-\iint \ln |z-t| d \nu(t) d \mu(z) .
$$

With these notations the entropy (2) is equivalently rewritten as

$$
E_{n}(\nu)=S\left(\nu_{n}, \nu\right)=-2 \ln \gamma_{n}+2 n I\left(\mu_{n}, \nu_{n}\right) .
$$

In particular, from classical Jensen's inequality for integrals, it follows immediately that if both $\mu$ and $\nu$ are unit measures on $\Delta$, then $S(\mu, \nu) \leq 0$, with equality if and only if $\mu=\nu$. Hence,

$$
E_{n}(\nu) \leq 0,
$$

with equality if and only if $n=0$.

Aptekarev et al. 1] considered two subfamilies of the usual Szegö class of weights on $\Delta$, namely the Jacobi weights and the Bernstein-Szegő class (weights being bounded above, bounded away from zero, and satisfying a Dini-Lipschitz condition). In this last case it is known that the asymptotic formula for the orthogonal polynomials $p_{n}$ holds uniformly in $\Delta$, as $n$ tends to infinity. With these assumptions it has been proved in [1] that

$$
\lim _{n \rightarrow \infty} E_{n}(w)=S(\rho, w)+\log (2)-1,
$$

where

$$
\rho(x)=1 /\left(\pi \sqrt{1-x^{2}}\right)
$$


denotes the Chebyshev unit weight on $\Delta$. We are concerned here with the problem whether a weaker form of this equality holds in the Szegö class of weights. We will show that the right-hand side of (5) is actually an asymptotic upper bound for the entropy $E_{n}(w)$ when the weight $w$ satisfies the Szegó condition (see assumption (6) below). Furthermore, the expression (2) for the entropy can be naturally split into two functionals, which have simple asymptotic behaviors when $w$ belongs to the Bernstein class (see Proposition प). The situation with the Bernstein class is in a sense optimal: the corresponding limits provide asymptotic upper bounds for $w$ in the whole Szegö class. We also give conditions for the entropy and the two functionals to tend to their upper bounds as the degree $n$ becomes large.

Finally, we must mention that in the case of an unbounded support of the weight of orthogonality interesting results concerning the asymptotics of the $E_{n}$ and related functionals have been obtained recently in 10.

\section{Statements of results}

The weighted $L^{p}$ norm of a function $f$ with respect to a weight $k$ on $\Delta$ will be denoted by

$$
\|f\|_{L^{p}(k)}=\left(\int_{\Delta}|f(x)|^{p} k(x) d x\right)^{1 / p}, \quad 1 \leq p \leq \infty .
$$

We will simply write $L^{p}$ when $k \equiv 1$ on $\Delta$.

Though our main interest in this paper lies in the Szegö class of weights, some other classes appear at different places. We recall the definitions of these classes now.

The Erdös-Turan class $\mathcal{E} \mathcal{T}$ consists of weights $w \in L^{1}$ such that $w>0$ almost everywhere on $\Delta$.

The Szegö class $\mathcal{S}$ consists of weights $w \in L^{1}$ such that

$$
\log \left(w_{0}\right) \in L^{1}(\rho),
$$

where

$$
w_{0}(x):=w(x) / \rho(x)=\pi \sqrt{1-x^{2}} w(x)
$$

denotes the trigonometric weight corresponding to $w$. The fact that $w_{0} \in L^{1}(\rho)$ implies $\log ^{+}\left(w_{0}\right) \in L^{1}(\rho)$, where, as usual, we denote

$$
\log ^{+}(x)=\max \{\log (x), 0\}, \quad x>0 .
$$

Hence, condition (6) is actually equivalent to

$$
S(\rho, w)=\int_{\Delta} \log \left(w_{0}(x)\right) \rho(x) d x>-\infty .
$$

Note that [6] and (7) can equivalently be rewritten as $\log (w) \in L^{1}(\rho)$ and

$$
\int_{\Delta} \log (w(x)) \rho(x) d x>-\infty
$$

respectively.

Finally the Bernstein class $\mathcal{B}$ consists of weights $w$ such that $w_{0}$ is given by the reciprocal of a positive polynomial on $\Delta$. As it is well-known, the class $\mathcal{B}$ is an important class useful for establishing asymptotic 
properties in the Szegö theory of orthogonal polynomials. Obviously, one has the following inclusions $\mathcal{B} \subset \mathcal{S} \subset \mathcal{E} \mathcal{T}$.

We will also use the notations:

$$
f_{n}(x):=p_{n}(x) \sqrt{w_{0}(x)}
$$

and for $M>0$,

$$
\Delta_{n}(M):=\left\{x \in \Delta:\left|f_{n}(x)\right| \geq M\right\} .
$$

One of the main results of the paper is:

Theorem 1 Assume that the weight $w$ belongs to the Szegö class $\mathcal{S}$. Then, for all $M>\sqrt{2}$,

$$
E_{n}(w)=S(\rho, w)+\log (2)-1-\int_{\Delta_{n}(M)} p_{n}^{2}(x) \log ^{+}\left(p_{n}^{2}(x)\right) w(x) d x+o(1), \quad n \rightarrow \infty .
$$

As a simple consequence of the above formula, we obtain the following asymptotic upper bound together with necessary and sufficient conditions for equality.

Corollary 1 Assume that the weight $w$ belongs to the Szego" class $\mathcal{S}$. Then the following asymptotic upper bound for the entropy holds :

$$
\limsup _{n \rightarrow \infty} E_{n}(w) \leq S(\rho, w)+\log (2)-1 .
$$

Moreover, for a subsequence $n \in \Lambda \subset \mathbb{N}$,

$$
\lim _{n \in \Lambda} E_{n}(w)=S(\rho, w)+\log (2)-1,
$$

if and only if there exists a constant $M>\sqrt{2}$, such that

$$
\lim _{n \in \Lambda} \int_{\Delta_{n}(M)} p_{n}^{2}(x) \log ^{+}\left(p_{n}^{2}(x)\right) w(x) d x=0 .
$$

In this case (13) is valid for for all $M>\sqrt{2}$.

Furthermore, 13) holds if there exists $\varepsilon>0$ such that either

$$
\sup _{n \in \Lambda} \int_{\Delta}\left(\log ^{+}\left(p_{n}^{2}(x)\right)\right)^{1+\varepsilon} p_{n}^{2}(x) w(x) d x<\infty \quad \text { or } \quad \sup _{n \in \Lambda} \int_{\Delta}\left(p_{n}^{2}(x)\right)^{1+\varepsilon} w(x) d x<\infty .
$$

Remark 1 Notice that the findings of [1] on Bernstein-Szegő polynomials are included in Corollary [1] since for $w \in \mathcal{B}, \log \left(w_{0}\right)$ is bounded and the $f_{n}$ are uniformly bounded in $[-1,1]$. In contrast, the case of Jacobi polynomials requires some extra considerations. One knows that for the orthonormal Jacobi polynomials there exists a constant $c$ such that for $n \geq 0$ and $x \in[-1,1]$,

$$
\left|P_{n}^{(\alpha, \beta)}(x)\right| \cdot\left(\sqrt{1-x}+\frac{1}{n}\right)^{\alpha+1 / 2}\left(\sqrt{1+x}+\frac{1}{n}\right)^{\beta+1 / 2} \leq c / \sqrt{\pi} .
$$

Taking into account that here $w_{0}(x)=\pi(1-x)^{\alpha+1 / 2}(1+x)^{\beta+1 / 2}$, we find that for $p_{n}=P_{n}^{(\alpha, \beta)}$,

$$
\left(p_{n}(x)\right)^{2+\varepsilon} w_{0}(x) \leq c \sqrt{\pi}\left(\frac{1-x}{(\sqrt{1-x}+1 / n)^{2+\varepsilon}}\right)^{\alpha+1 / 2}\left(\frac{1+x}{(\sqrt{1+x}+1 / n)^{2+\varepsilon}}\right)^{\beta+1 / 2}
$$

and the second condition in (14) is satisfied. 
Remark 2 An inequality weaker than (11) is a straightforward consequence of the asymptotic behavior of the measures $\nu_{n}$. Indeed, if $w \in \mathcal{E} \mathcal{T}$, we know from Rakhmanov's Theorem 13] that $d \nu_{n}(x) \rightarrow \rho(x) d x$ as $n \rightarrow \infty$ in the weak-* topology. It follows from the weak upper semicontinuity of the mutual entropy [8. Corollary 5.3] that $\lim \sup E_{n}(w)=\lim \sup S\left(\nu_{n}, w\right) \leq S(\rho, w)$. In particular, it shows that if the weight $w$ is in $\mathcal{E} \mathcal{T} \backslash \mathcal{S}$,

$$
\lim _{n \rightarrow \infty} E_{n}(w)=-\infty .
$$

Nevertheless, it seems that a semicontinuity argument for the entropy does not allow to explain the additional term $\log (2)-1$ occurring on the right-hand side of (12).

The information entropy for Chebyshev polynomials orthonormal with respect to $\rho$ has been computed in [4] 16]:

$$
E_{n}(\rho)=\log (2)-1 \quad \text { for } n \geq 1 .
$$

Intuitively, Chebyshev polynomials are the most "uniformly" distributed polynomials, both for each $n$ and asymptotically as $n \rightarrow \infty$. This fact is formally set in the next corollary.

\section{Corollary 2 If}

$$
\limsup _{n \rightarrow \infty} E_{n}(w) \geq \log (2)-1,
$$

then $w=\rho$ and $E_{n}(w)=\log (2)-1, n \geq 1$.

The proof is a simple consequence of inequality (11). Indeed, from this inequality, we see that (16) can only happen if $S(\rho, w)=0$ that is $\rho=w$.

Now we exploit the connection between the entropy $E_{n}(w)$ and the mutual energy $I\left(\mu_{n}, \nu_{n}\right)$ given in (44). It is well known that in the class $\mathcal{E} \mathcal{T}$ both $\mu_{n}$ and $\nu_{n}$ tend (as $\left.n \rightarrow \infty\right)$ to the Chebyshev (equilibrium) distribution given by the weight $\rho$ on $\Delta$. In particular, from the convexity properties of the mutual energy it follows that

$$
\lim _{n \rightarrow \infty} I\left(\mu_{n}, \nu_{n}\right)=I(\rho, \rho)=\log (2) .
$$

What is more surprising is that the next term of the asymptotic expansion of $I\left(\mu_{n}, \nu_{n}\right)$ also exhibits a "universal" behavior, in the sense that it does not depend on the choice of the weight $w$. Namely, if the entropy $E_{n}(w)$ satisfies (12), then the following result is a direct consequence of (4) and the well known asymptotic behavior of the leading coefficient of $p_{n}$ (see (29) below):

Corollary 3 Assume $w$ is a weight in the Szegö class $\mathcal{S}$ and condition 113) is satisfied. Then the mutual energy $I\left(\mu_{n}, \nu_{n}\right)$ has the following asymptotic expansion:

$$
I\left(\mu_{n}, \nu_{n}\right)=\log (2)-\frac{1}{2 n}+o\left(\frac{1}{n}\right), \quad n \in \Lambda, \quad n \rightarrow \infty .
$$

This remarkable fact certainly deserves further study.

Another aim of the paper is to study two related functionals $F_{n}$ and $G_{n}$, whose sum equals the entropy,

$$
E_{n}(w)=F_{n}(w)+G_{n}(w),
$$


and which are defined by

$$
F_{n}(w)=-\int_{\Delta} \log \left(p_{n}^{2}(x) w_{0}(x)\right) p_{n}^{2}(x) w(x) d x=S\left(f_{n}^{2} \rho, \rho\right),
$$

and

$$
G_{n}(w)=\int_{\Delta} \log \left(w_{0}(x)\right) p_{n}^{2}(x) w(x) d x=-S\left(p_{n}^{2} w, p_{n}^{2} \rho\right) .
$$

We will see that the functional $F_{n}$ also exhibits a "universal" behavior, while $G_{n}$ is sensitive to a particular choice of the weight $w$, and is related naturally with the mutual entropy $S(\rho, w)$. Functionals $F_{n}$ and $G_{n}$ have a particularly nice behavior for $w$ in the Bernstein class $\mathcal{B}$ :

Proposition 1 Let $S$ be a polynomial of degree $2 N(N \geq 0)$ such that $S(x)>0$ for $x \in \Delta$, and assume that the orthogonality weight satisfies

$$
w_{0}(x)=\frac{1}{S(x)}, \quad x \in \Delta
$$

Then

$$
F_{n}(w)=\log (2)-1 \quad \text { for } n>N
$$

Moreover,

$$
\lim _{n \rightarrow \infty} G_{n}(w)=S(\rho, w),
$$

and this limit takes place with a geometric rate. Consequently, the same holds true for the limit in (5).

The conjecture that constant entropy $E_{n}(w)$ is a (yet another) characterization of Chebyshev polynomials (cf. (15)) belongs to L. Golinsky. We were able to prove it in the Bernstein class $\mathcal{B}$.

Proposition 2 Let $w \in \mathcal{B}$ such that $E_{n}(w)$ is constant for all sufficiently large $n$. Then $w=\rho$.

Since Bernstein weights are suitable as approximation tool for the whole Szegö class, we could expect the asymptotic behavior from Proposition 1 to hold in a more general setting. Nevertheless, the behavior of the entropy, as well as the behavior of the two functionals $F_{n}$ and $G_{n}$, is extremely sensitive to the growth of $p_{n}^{2} w$, which may affect convergence. In general, the following expression for the first functional $F_{n}$ holds true:

Theorem 2 Assume the weight $w$ belongs to the Szegö class $\mathcal{S}$. Then, for all $M>\sqrt{2}$,

$$
F_{n}(w)=\log (2)-1-\int_{\Delta_{n}(M)} \log \left(f_{n}^{2}(x)\right) f_{n}^{2}(x) \rho(x) d x+o(1), \quad n \rightarrow \infty .
$$

Again, as a simple consequence of the above formula, we get the

Corollary 4 Assume the weight $w$ belongs to the Szegö class $\mathcal{S}$. Then, the following asymptotic upper bound for $F_{n}$ holds:

$$
\limsup _{n \rightarrow \infty} F_{n}(w) \leq \log (2)-1
$$


Moreover, for a subsequence $n \in \Lambda \subset \mathbb{N}$,

$$
\lim _{n \in \Lambda} F_{n}(w)=\log (2)-1
$$

if and only if there exists a constant $M>\sqrt{2}$, such that

$$
\lim _{n \in \Lambda} \int_{\Delta_{n}(M)} f_{n}^{2}(x) \log \left(f_{n}^{2}(x)\right) \rho(x) d x=0,
$$

for $f_{n}$ and $\Delta_{n}(M)$ defined in (8) and (9), respectively. In this case, 24) is valid for every $M>\sqrt{2}$.

Furthermore, 24) holds if there exists an $\varepsilon>0$ such that either

$$
\sup _{n \in \Lambda} \int_{\Delta}\left(\log ^{+}\left(f_{n}^{2}(x)\right)\right)^{1+\varepsilon} f_{n}^{2}(x) \rho(x) d x<\infty \quad \text { or } \quad \sup _{n \in \Lambda} \int_{\Delta}\left(f_{n}^{2}(x)\right)^{1+\varepsilon} \rho(x) d x<\infty .
$$

Remark 3 The method of proof of Theorem 2 can be applied to larger classes of weights. In fact, we only need an $L^{2}$ asymptotics of the polynomials $p_{n}$ on the support $\Delta$ of the measure $\nu$, and that has been extended beyond the Szegö class. For instance, using our technique we can prove that (11) is valid for weights $w \in \mathcal{F}($ dini $)$, introduced in 9 .

Remark 4 Apparently, a necessary condition for (25) is that $w_{0} \log \left(w_{0}\right) \in L^{1}(\rho)$ (cf. with (6)). If $\log \left(w_{0}\right) \in L^{\infty}$ then there is equivalence between conditions (13) and (24), and between (14) and (25), respectively.

Concerning the second functional $G_{n}$, we use a result from [11 to deduce the following proposition.

Proposition 3 Assume the weight $w$ belongs to the Szegö class $\mathcal{S}$ and $\log ^{+}\left(w_{0}\right) \in L^{\infty}$, then

$$
\limsup _{n \rightarrow \infty} G_{n}(w) \leq S(\rho, w)=\int_{\Delta} \log \left(w_{0}(x)\right) \rho(x) d x .
$$

Similarly, assume that $\log ^{-}\left(w_{0}\right) \in L^{\infty}$, then

$$
\liminf _{n \rightarrow \infty} G_{n}(w) \geq S(\rho, w)=\int_{\Delta} \log \left(w_{0}(x)\right) \rho(x) d x .
$$

Hence, if $\log \left(w_{0}\right) \in L^{\infty}$, then

$$
\lim _{n \rightarrow \infty} G_{n}(w)=S(\rho, w) .
$$

Furthermore, if the weight $w$ belongs to the set $\mathcal{E} \mathcal{T} \backslash \mathcal{S}$, the assumption $\log ^{+}\left(w_{0}\right) \in L^{\infty}$ still implies inequality (26). In this case, (26) simplifies to $\lim _{n \rightarrow \infty} G_{n}(w)=-\infty$.

\section{Proofs of Theorems 1 and 2 , Corollaries 1 and 4}

Before entering the proofs of our results, let us state two preliminary lemmas. The first one is borrowed from [1]. 
Lemma 1 [1. Lemma 2.1] Let $g$ be a continuous function on $\mathbb{R}, g(\theta+\pi)=g(\theta), f \in L^{1}([0, \pi])$, and let $\gamma(\theta)$ be a function that is mesurable and almost everywhere finite on $[0, \pi]$. Then, as $n \rightarrow \infty$,

$$
\int_{0}^{\pi} g(n \theta+\gamma(\theta)) f(\theta) d \theta \rightarrow \frac{1}{\pi} \int_{0}^{\pi} g(\theta) d \theta \int_{0}^{\pi} f(\theta) d \theta .
$$

As remarked in [1], when $\gamma(\theta)=0$ and $g \in L^{\infty}[0, \pi]$, the statement of the lemma becomes a well-known result of Fejer, cf. [2, Chapter I, §20]. if

As second main ingredient in our proofs let us recall the Szegó asymptotics for $f_{n}(x)=\sqrt{w_{0}(x)} p_{n}(x)$ :

$$
g_{n}(x)=\sqrt{2} \cos (n \arccos x+\gamma(x)),
$$

where

$$
\gamma(x)=\frac{1}{2 \pi} \int_{\Delta} \frac{\log w_{0}(x)-\log w_{0}(t)}{x-t} \sqrt{\frac{1-x^{2}}{1-t^{2}}} d t
$$

is the harmonic conjugate function to $\log w_{0}$, then in the Szegö class $\mathcal{S}$, one has

$$
\lim _{n \rightarrow \infty}\left\|f_{n}-g_{n}\right\|_{L^{2}(\rho)}=0
$$

and

$$
\lim _{n \rightarrow \infty} \log \left(\frac{\gamma_{n}}{2^{n}}\right)=-\frac{1}{2}(\log (2)+S(\rho, w)) .
$$

The mutual entropy in the right hand side of (29) is known as the Szegö constant for the weight $w$. Since the entropy integral is very sensitive to the growth of $f_{n}^{2}=p_{n}^{2} w_{0}$, the following lemma will be useful; roughly speaking, it shows that the subsets $\Delta_{n}(M)$, defined in (9), have no influence on the $L^{2}$ asymptotics 28:

Lemma 2 For $w \in \mathcal{S}$,

$$
\lim _{n \rightarrow \infty} \int_{\Delta_{n}(M)} \rho(x) d x=0 .
$$

for every $M>\sqrt{2}$. Furthermore, let $\widetilde{f}_{n}, n \geq 0$, be the sequence of truncated functions

$$
\widetilde{f}_{n}(x):= \begin{cases}f_{n}(x) & \text { for } x \in \Delta \backslash \Delta_{n}(M) \\ 1 & \text { for } x \in \Delta_{n}(M)\end{cases}
$$

Then

$$
\lim _{n \rightarrow \infty}\left\|\widetilde{f}_{n}-g_{n}\right\|_{L^{2}(\rho)}=0 .
$$

Proof. Observe first that by Cauchy-Schwarz inequality,

$$
\int_{\Delta}\left|f_{n}^{2}(x)-g_{n}^{2}(x)\right| \rho(x) d x \leq\left\|f_{n}+g_{n}\right\|_{L^{2}(\rho)} \cdot\left\|f_{n}-g_{n}\right\|_{L^{2}(\rho)} \leq\left(\left\|f_{n}\right\|_{L^{2}(\rho)}+\left\|g_{n}\right\|_{L^{2}(\rho)}\right) \cdot\left\|f_{n}-g_{n}\right\|_{L^{2}(\rho)},
$$

so that

$$
\int_{\Delta}\left|f_{n}^{2}(x)-g_{n}^{2}(x)\right| \rho(x) d x \leq(1+\sqrt{2})\left\|f_{n}-g_{n}\right\|_{L^{2}(\rho)} .
$$


Now we can show that the Chebyshev (and hence, Lebesgue) measure of $\Delta_{n}(M)$ is asymptotically vanishing: by (33),

$$
\begin{aligned}
\left(M^{2}-2\right) \int_{\Delta_{n}(M)} \rho(x) d x & \leq \int_{\Delta_{n}(M)}\left(f_{n}^{2}(x)-2\right) \rho(x) d x \leq \int_{\Delta_{n}(M)}\left|f_{n}^{2}(x)-g_{n}^{2}(x)\right| \rho(x) d x \\
& \leq(1+\sqrt{2})\left\|f_{n}-g_{n}\right\|_{L^{2}(\rho)},
\end{aligned}
$$

the right-hand side tending to zero as $n \rightarrow \infty$ by (28); this proves (30). Moreover, since $\left|\widetilde{f}_{n}(x)\right|=1$ and $\left|g_{n}(x)\right| \leq \sqrt{2}$ for $x \in \Delta_{n}(M)$, we have by 33)

$$
\begin{aligned}
\left\|\tilde{f}_{n}-g_{n}\right\|_{L^{2}(\rho)}^{2} & =\int_{\Delta \backslash \Delta_{n}(M)}\left|f_{n}(x)-g_{n}(x)\right|^{2} \rho(x) d x+\int_{\Delta_{n}(M)}\left|\tilde{f}_{n}(x)-g_{n}(x)\right|^{2} \rho(x) d x \\
& \leq(1+\sqrt{2})\left\|f_{n}-g_{n}\right\|_{L^{2}(\rho)}+3 \int_{\Delta_{n}(M)} \rho(x) d x .
\end{aligned}
$$

It remains to use (28) and (30) to see that (32) is satisfied.

\subsection{Proof of Theorem [1}

Fix arbitrary $M>\sqrt{2}$ and let $\Delta_{n}(M)$ and $\widetilde{f}$ be as defined in (91) and (31), respectively. We write the entropy as

$$
E_{n}(w)=S\left(f_{n}^{2} \rho, w\right)=S\left(g_{n}^{2} \rho, w\right)+\left[S\left(\widetilde{f}_{n}^{2} \rho, w\right)-S\left(g_{n}^{2} \rho, w\right)\right]+\left[S\left(f_{n}^{2} \rho, w\right)-S\left(\widetilde{f}_{n}^{2} \rho, w\right)\right] .
$$

In three steps let us prove that the first term on the right has as limit the first three terms in the righthand side of (10), the second term tends to 0 , and the third term is asymptotically negative and related to the integral in (10).

Let

$$
\mathcal{R}(y)=y^{2} \log \left(y^{2}\right), \quad y \in \mathbb{R} .
$$

From Lemma 1 we get

$$
\begin{aligned}
& \lim _{n \rightarrow \infty} S\left(g_{n}^{2} \rho, w\right) \\
& =-\lim _{n \rightarrow \infty} \int_{0}^{\pi} \mathcal{R}\left(g_{n}(\cos (\theta))\right) \frac{d \theta}{\pi}+\lim _{n \rightarrow \infty} \int_{0}^{\pi} \log \left(w_{0}(\cos (\theta))\right) g_{n}^{2}(\cos (\theta)) \frac{d \theta}{\pi} \\
& =-\int_{0}^{\pi} \mathcal{R}(\sqrt{2} \cos (\theta)) \frac{d \theta}{\pi}+\int_{0}^{\pi} 2 \cos ^{2}(\theta) \frac{d \theta}{\pi} \int_{0}^{\pi} \log \left(w_{0}(\cos (\theta))\right) \frac{d \theta}{\pi} \\
& =E_{1}(\rho)+S(\rho, w)=\log (2)-1+S(\rho, w) .
\end{aligned}
$$

Hence the first term on the right-hand side of (34) has the required limit. The second term in (34) can be written as

$$
S\left(\widetilde{f}_{n}^{2} \rho, w\right)-S\left(g_{n}^{2} \rho, w\right)=\int_{\Delta}\left[\mathcal{R}\left(\frac{\widetilde{f}_{n}(x)}{\sqrt{w_{0}(x)}}\right)-\mathcal{R}\left(\frac{g_{n}(x)}{\sqrt{w_{0}(x)}}\right)\right] w(x) d x .
$$


Recall that both $\widetilde{f}_{n}$ and $g_{n}$ are uniformly bounded on $\Delta$ by $M$, and hence for $x \in \Delta$

$$
\left|\mathcal{R}\left(\frac{\widetilde{f}_{n}(x)}{\sqrt{w_{0}(x)}}\right)\right| w_{0}(x) \leq\left|\mathcal{R}\left(\widetilde{f}_{n}(x)\right)\right|+\left|\log \left(w_{0}(x)\right)\right| \widetilde{f}_{n}^{2}(x) \leq M^{2} \log M^{2}+M^{2}\left|\log \left(w_{0}(x)\right)\right|=: h(x),
$$

where $h \in L^{1}(\rho)$ by assumption [6]. Similarly,

$$
\left|\mathcal{R}\left(\frac{g_{n}(x)}{\sqrt{w_{0}(x)}}\right)\right| w_{0}(x) \leq h(x), \quad x \in \Delta .
$$

The integral in (36) will be split into two parts depending on whether $w_{0}$ is small or large. Fix an arbitrary $0<\varepsilon<1$; by the monotone convergence theorem there exists a constant $C=C(\varepsilon)$ such that

$$
0 \leq \int_{h(x)>C} h(x) \rho(x) d x=\int_{\Delta} h(x) \rho(x) d x-\int_{h(x) \leq C} h(x) \rho(x) d x<\varepsilon .
$$

Defining $\tau:=M^{2} \exp \left(-C / M^{2}\right)$ we see that $w_{0}(x)<\tau$ implies that $h(x)>C$, and hence

$$
\left|\int_{w_{0}(x)<\tau}\left[\mathcal{R}\left(\frac{\widetilde{f}_{n}(x)}{\sqrt{w_{0}(x)}}\right)-\mathcal{R}\left(\frac{g_{n}(x)}{\sqrt{w_{0}(x)}}\right)\right] w(x) d x\right| \leq 2 \int_{w_{0}(x)<\tau} h(x) \rho(x) d x \leq 2 \varepsilon .
$$

On the other hand, if $w_{0}(x) \geq \tau$, then

$$
\left|\frac{\tilde{f}_{n}(x)}{\sqrt{w_{0}(x)}}\right| \leq \frac{M}{\sqrt{w_{0}(x)}} \leq \frac{M}{\sqrt{\tau}}=e^{C /\left(2 M^{2}\right)}=: C_{1},
$$

and the same inequality is valid for $g_{n} / \sqrt{w_{0}}$. Taking into account that $\mathcal{R}$ is smooth,

$$
\begin{aligned}
& \left|\mathcal{R}\left(\frac{\tilde{f}_{n}(x)}{\sqrt{w_{0}(x)}}\right)-\mathcal{R}\left(\frac{g_{n}(x)}{\sqrt{w_{0}(x)}}\right)\right| \leq \max _{|y| \leq C_{1}}\left|\mathcal{R}^{\prime}(y)\right|\left|\frac{\widetilde{f}_{n}(x)}{\sqrt{w_{0}(x)}}-\frac{g_{n}(x)}{\sqrt{w_{0}(x)}}\right| \\
& \leq \max _{|y| \leq C_{1}}\left|2 y\left(1+\log \left(y^{2}\right)\right)\right|\left|\frac{\widetilde{f}_{n}(x)}{\sqrt{w_{0}(x)}}-\frac{g_{n}(x)}{\sqrt{w_{0}(x)}}\right| \\
& \leq C_{2}\left|\frac{\widetilde{f}_{n}(x)}{\sqrt{w_{0}(x)}}-\frac{g_{n}(x)}{\sqrt{w_{0}(x)}}\right|
\end{aligned}
$$

with $C_{2}:=\max \left\{4 e^{-3 / 2}, 2 C_{1}\left(1+\log \left(C_{1}^{2}\right)\right)\right\}$. Hence, using the Cauchy-Schwarz inequality,

$$
\begin{aligned}
\left|\int_{w_{0}(x) \geq \tau}\left[\mathcal{R}\left(\frac{\tilde{f}_{n}(x)}{\sqrt{w_{0}(x)}}\right)-\mathcal{R}\left(\frac{g_{n}(x)}{\sqrt{w_{0}(x)}}\right)\right] w(x) d x\right| & \leq C_{2}\left\|\left(\tilde{f}_{n}-g_{n}\right) \sqrt{w_{0}(x)}\right\|_{L^{1}(\rho)} \\
& \leq C_{2}\left\|\tilde{f}_{n}-g_{n}\right\|_{L^{2}(\rho)},
\end{aligned}
$$

which by (32) tends to 0 as $n \rightarrow \infty$. Taking into account that $\varepsilon \in(0,1)$ was chosen arbitrary, we conclude that

$$
S\left(\widetilde{f}_{n}^{2} \rho, w\right)-S\left(g_{n}^{2} \rho, w\right) \longrightarrow 0, \quad n \rightarrow \infty .
$$


Thus, for establishing the expression for the entropy in Theorem 1 it only remains to examine the last bracket on the right-hand side of (34). Notice that since $\widetilde{f}_{n}=f_{n}$ on $\Delta \backslash \Delta_{n}(M)$,

$$
\begin{aligned}
S\left(f_{n}^{2} \rho, w\right) & -S\left(\widetilde{f}_{n}^{2} \rho, w\right) \\
= & -\int_{\Delta_{n}(M)} p_{n}^{2}(x) \log \left(p_{n}^{2}(x)\right) w(x) d x+\int_{\Delta_{n}(M)} \log \left(\frac{1}{w_{0}(x)}\right) \rho(x) d x \\
= & -\int_{\Delta_{n}(M)} p_{n}^{2}(x) \log ^{+}\left(p_{n}^{2}(x)\right) w(x) d x+\int_{\widetilde{\Delta}_{n}(M)} p_{n}^{2}(x)\left|\log \left(p_{n}^{2}(x)\right)\right| w(x) d x \\
& -\int_{\Delta_{n}(M)} \log \left(w_{0}(x)\right) \rho(x) d x
\end{aligned}
$$

where

$$
\widetilde{\Delta}_{n}(M)=\left\{x \in \Delta_{n}(M): p_{n}^{2}(x)<1\right\} \subset \Delta_{n}(M) .
$$

Observing that, for $p_{n}(x)^{2} \leq 1$, we have $0 \leq p_{n}^{2}(x)\left|\log \left(p_{n}^{2}(x)\right)\right| \leq 1$, we obtain

$$
0 \leq \int_{\widetilde{\Delta}_{n}(M)} p_{n}^{2}(x)\left|\log \left(p_{n}^{2}(x)\right)\right| w(x) d x \leq \int_{\Delta_{n}(M)} w(x) d x=\int_{\Delta_{n}(M)} w_{0}(x) \rho(x) d x .
$$

Since $w_{0} \in L^{1}(\rho), \log \left(w_{0}\right) \in L^{1}(\rho)$, by the absolute continuity of the Lebesgue integral, relation (30) implies that

$$
\lim _{n \rightarrow \infty} \int_{\Delta_{n}(M)} w_{0}(x) \rho(x) d x=0, \quad \text { and } \quad \lim _{n \rightarrow \infty} \int_{\Delta_{n}(M)} \log \left(w_{0}(x)\right) \rho(x) d x=0,
$$

showing that

$$
S\left(f_{n}^{2} \rho, w\right)-S\left(\widetilde{f}_{n}^{2} \rho, w\right)=-\int_{\Delta_{n}(M)} p_{n}^{2}(x) \log ^{+}\left(p_{n}^{2}(x)\right) w(x) d x+o(1), \quad n \rightarrow \infty .
$$

Hence, gathering (35), (37), and (39) in (34), we get (10).

\subsection{Proof of Corollary 1}

Since

$$
\int_{\Delta_{n}(M)} p_{n}^{2}(x) \log ^{+}\left(p_{n}^{2}(x)\right) w(x) d x \geq 0,
$$

relation (11) is a trivial consequence of Theorem 10 Suppose now that (13) holds for some $M>\sqrt{2}$, then (12) follows immediately from (10). Conversely, if (12) is true then it follows from Theorem (1) that (13) holds for all $M>\sqrt{2}$.

In order to prove that (14) is sufficient for (13), notice that, by Hölder's inequality,

$$
\begin{aligned}
& \int_{\Delta_{n}(M)} p_{n}^{2}(x) \log ^{+}\left(p_{n}^{2}(x)\right) w(x) d x \\
& \leq\left(\int_{\Delta_{n}(M)} p_{n}^{2}(x)\left(\log ^{+}\left(p_{n}^{2}(x)\right)\right)^{1+\epsilon} w(x) d x\right)^{\frac{1}{1+\epsilon}}\left(\int_{\Delta_{n}(M)} p_{n}^{2}(x) w(x) d x\right)^{1-\frac{1}{1+\epsilon}} .
\end{aligned}
$$


Furthermore,

$$
\begin{aligned}
& \int_{\Delta_{n}(M)} p_{n}^{2}(x) w(x) d x \leq \int_{\Delta_{n}(M)}\left[f_{n}^{2}(x)-g_{n}^{2}(x)\right] \rho(x) d x+\int_{\Delta_{n}(M)} g_{n}^{2}(x) \rho(x) d x \\
& \leq \int_{\Delta_{n}(M)}\left[f_{n}^{2}(x)-g_{n}^{2}(x)\right] \rho(x) d x+2 \int_{\Delta_{n}(M)} \rho(x) d x \\
& \leq(1+\sqrt{2})\left\|f_{n}-g_{n}\right\|_{L^{2}(\rho)}+2 \int_{\Delta_{n}} \rho(x) d x=o(1), \quad n \rightarrow \infty
\end{aligned}
$$

where we have used (28), (30) and (33).

If we assume that the first condition in (14) holds, then the first factor on the right hand side of [40] is uniformly bounded in $n$, and (13) follows.

Finally, notice that the second condition in (14) implies the first one since $\log ^{+}(z) \leq z$ for $z \geq 0$, and hence

$$
\left(\log ^{+}(y)\right)^{1+\varepsilon}=\left(\frac{1+\varepsilon}{\varepsilon}\right)^{1+\varepsilon}\left(\log ^{+}\left(y^{\frac{\varepsilon}{1+\varepsilon}}\right)\right)^{1+\varepsilon} \leq\left(\frac{1+\varepsilon}{\varepsilon}\right)^{1+\varepsilon} y^{\varepsilon}, \quad y \geq 0 .
$$

\subsection{Proof of Theorem 2}

Our proof for Theorem 2 follows closely the arguments of the proof of Theorem 1 but some parts simplify. As before let $\mathcal{R}(y)=y^{2} \log \left(y^{2}\right), y \in \mathbb{R}$, and fix $M>\sqrt{2}$. We write the functional as follows:

$$
\begin{aligned}
F_{n}(w)= & \int_{\Delta}\left[-\mathcal{R}\left(g_{n}(x)\right)\right] \rho(x) d x \\
& +\int_{\Delta}\left[\mathcal{R}\left(g_{n}(x)\right)-\mathcal{R}\left(\widetilde{f}_{n}(x)\right)\right] \rho(x) d x+\int_{\Delta}\left[\mathcal{R}\left(\widetilde{f}_{n}(x)\right)-\mathcal{R}\left(f_{n}(x)\right)\right] \rho(x) d x .
\end{aligned}
$$

Here the first integral on the right-hand side of (41) has the limit $E_{1}(\rho)=\log (2)-1$ by Lemma 1 The last one can be written as

$$
\int_{\Delta}\left[\mathcal{R}\left(\widetilde{f}_{n}(x)\right)-\mathcal{R}\left(f_{n}(x)\right)\right] \rho(x) d x=-\int_{\Delta_{n}(M)} \log \left(f_{n}^{2}(x)\right) f_{n}^{2}(x) \rho(x) d x \leq 0,
$$

the right-hand side coinciding with the integral in (21). Thus Theorem 2 follows by showing that the second integral on the right-hand side of (41) is asymptotically vanishing. Recalling that $\left|\widetilde{f}_{n}(x)\right|$ and $\left|g_{n}(x)\right|$ are uniformly bounded by $M$ for all $n \geq 0$ and $x \in \Delta$, we obtain

$$
\begin{aligned}
& \left.\left|\int_{\Delta}\left[\mathcal{R}\left(g_{n}(x)\right)-\mathcal{R}\left(\widetilde{f}_{n}(x)\right)\right] \rho(x) d x\right| \leq \max _{y \in[-M, M]}\left|\mathcal{R}^{\prime}(y)\right| \int_{\Delta} \mid g_{n}(x)-\widetilde{f}_{n}(x)\right) \mid \rho(x) d x \\
& \leq M^{2}\left(1+\log M^{2}\right)\left\|g_{n}-\widetilde{f}_{n}\right\|_{L^{1}(\rho)} \leq M^{2}\left(1+\log M^{2}\right)\left\|g_{n}-\widetilde{f}_{n}\right\|_{L^{2}(\rho)},
\end{aligned}
$$

the term on the right tending to zero as $n \rightarrow \infty$ by (32). 


\subsection{Proof of Corollary 4}

Since

$$
\int_{\Delta_{n}(M)} f_{n}^{2}(x) \log \left(f_{n}^{2}(x)\right) \rho(x) d x \geq 0,
$$

relation (22) is a trivial consequence of Theorem[2] Suppose now that (24) holds for some $M>\sqrt{2}$, then (23) follows immediately from (21). Conversely, if (23) is true then it follows from Theorem 2 that (24) holds for all $M>\sqrt{2}$.

In order to prove that the first condition in (25) (which clearly is weaker than the second one) is sufficient for (24), notice that, by Hölder's inequality,

$$
\begin{aligned}
& \int_{\Delta_{n}} f_{n}^{2}(x) \log ^{+}\left(f_{n}^{2}(x)\right) \rho(x) d x \\
& \leq\left(\int_{\Delta_{n}} f_{n}^{2}(x)\left(\log ^{+}\left(f_{n}^{2}(x)\right)\right)^{1+\varepsilon} \rho(x) d x\right)^{\frac{1}{1+\varepsilon}}\left(\int_{\Delta_{n}} f_{n}^{2}(x) \rho(x) d x\right)^{1-\frac{1}{1+\varepsilon}},
\end{aligned}
$$

and we may conclude as in the proof of Corollary 1 that the second factor in the right hand side tends to zero.

\section{Proofs of Propositions 1, 2, and 3.}

\subsection{Proof of Proposition 1}

Let us make the change of variables $x=(z+1 / z) / 2$. It is well known that since $S(x)>0$ on $\Delta$ we may write $S$ as

$$
S(x)=|q(z)|^{2}=q(z) q(1 / z)
$$

with $q$ a polynomial of degree $2 N$ with real coefficients having all its zeros outside the disk and $q(0)>0$. Moreover

$$
p_{n}(x)=\frac{1}{\sqrt{2}}\left(z^{n} q\left(z^{-1}\right)+z^{-n} q(z)\right)
$$

is the orthonormal polynomial of degree $n>N$ with respect to the Bernstein weight $\rho / S$. Introducing the Blaschke product

$$
B_{n}(z)=z^{2 n} q(1 / z) / q(z), \quad n \geq N,
$$

we find that

$$
p_{n}^{2}(x) w_{0}(x)=\frac{1}{2}\left|1+B_{n}(z)\right|^{2}=1+\frac{1}{2}\left(B_{n}(z)+B_{n}(1 / z)\right), \quad|z|=1 .
$$

Since, for $n>N, B_{n}(0)=0$, and $B_{n}$ is analytic in the disk, we have

$$
\begin{aligned}
\log (2)-F_{n}(w) & =\log (2)+\int \log \left(p_{n}^{2}(x) w_{0}(x)\right) p_{n}^{2}(x) w_{0}(x) \rho(x) d x \\
& =\frac{1}{2 \pi} \int_{|z|=1} \log \left(\left|1+B_{n}(z)\right|^{2}\right)\left[1+\frac{1}{2}\left(B_{n}(z)+B_{n}(1 / z)\right)\right]|d z| \\
& =\operatorname{Re}\left(\frac{1}{2 \pi i} \int_{|z|=1} \log \left(1+B_{n}(z)\right)\left[2+B_{n}(z)+B_{n}(1 / z)\right] \frac{d z}{z}\right) .
\end{aligned}
$$


Since $\left|B_{n}(z)\right|<1$ for $|z|<1$, the function $\log \left(1+B_{n}\right)\left[2+B_{n}\right]$ is holomorphic inside the disk and vanishes at the origin. Thus,

$$
\begin{aligned}
\log (2)-F_{n}(w) & =\operatorname{Re}\left(\frac{1}{2 \pi i} \int_{|z|=1} \log \left(1+B_{n}(z)\right) B_{n}(1 / z) \frac{d z}{z}\right) \\
& =\operatorname{Re}\left(\frac{1}{2 \pi i} \int_{|z|=1} \frac{\log \left(1+B_{n}(z)\right)}{B_{n}(z)} \frac{d z}{z}\right),
\end{aligned}
$$

where we have used that $B_{n}(1 / z)=1 / B_{n}(z)$. Observe that the last integrand is analytic in a neighborhood of the unit circle, and we can integrate along a smaller circle $|z|=r<1$, where $\left|B_{n}(z)\right|<1$. Replacing log by its uniformly convergent Taylor expansion we get finally that this integral equals 1 , which proves (19).

On the other hand, by a similar reasoning we have

$$
\begin{aligned}
G_{n}(w) & =\int_{-1}^{1} \log \left(w_{0}(x)\right) p_{n}(x)^{2} w(x) d x \\
& =-2 \operatorname{Re}\left(\frac{1}{2 \pi i} \int_{|z|=1} \log (q(z))\left[1+\frac{1}{2}\left(B_{n}(z)+B_{n}(1 / z)\right)\right]|d z|\right) \\
& =-2 \log (q(0))-\frac{1}{2 \pi i} \int_{|z|=1} \log (q(z)) B_{n}(1 / z) \frac{d z}{z} .
\end{aligned}
$$

Note that in the last expression of [47], taking the real part is not necessary since $q$ and $B_{n}$ are real functions. Integrating now along $|z|=R>1$, we observe that $\left|B_{n}(1 / z)\right|$ becomes there geometrically small, which yields a geometric rate of convergence for

$$
\lim _{n \rightarrow \infty} G_{n}(w)=-2 \log (q(0))=-\operatorname{Re} \frac{1}{\pi} \int_{|z|=1} \log (q(z)) \frac{d z}{z}=S(\rho, w),
$$

which proves 201.

\subsection{Proof of Proposition 2}

From the computations of $F_{n}(w)$ and $G_{n}(w)$ in the proof of Proposition 10 see (45) and (47), we know that $E_{n}(w)$ is constant for $n$ large, say $n>N_{0}>N$, if and only if

$$
\frac{1}{2 \pi i} \int_{|z|=1} \frac{B_{N}(1 / z) \log (q(z))}{z^{2 n-2 N}} \frac{d z}{z}=0, \quad n>N_{0},
$$

where the polynomial $q$ and the Blaschke product $B_{N}$ are defined by (42) and (44) respectively. Since $\log (q(z))$ is analytic in some neighborhood $\mathcal{U}$ of the unit disk, we may conclude that $\log (q(z)) B_{N}(1 / z)$ is meromorphic in $\mathcal{U}$, and thus can be written as

$$
B_{N}(1 / z) \log (q(z))=r(z)+f(z), \quad z \in \mathcal{U}
$$

where $r$ is a rational function such that $z^{2 N} q(1 / z) r(z)$ is a polynomial of degree at most $2 N-1$, and $f$ is analytic in $\mathcal{U}$. Since $r$ is analytic outside the unit disk and grows like at most $1 / z$ at infinity, we deduce

$$
\frac{1}{2 \pi i} \int_{|z|=1} \frac{r(z)}{z^{2 n-2 N}} \frac{d z}{z}=0, \quad n>N_{0}
$$


which implies, together with [48] and [49], that

$$
\frac{1}{2 \pi i} \int_{|z|=1} \frac{f(z)}{z^{2 n-2 N}} \frac{d z}{z}=0, \quad n>N_{0} .
$$

Hence, all sufficiently high even Taylor coefficients of $f$ vanish. As a consequence, $f(z)+f(-z)=P(z)$ is a polynomial, and

$$
B_{N}(1 / z) \log (q(z))+B_{N}(-1 / z) \log (q(-z))=r(z)+r(-z)+P(z), \quad|z| \leq 1 .
$$

Since the right-hand side of (50) is a rational function, the principle of analytic continuation applies, showing that (50) actually holds everywhere in $\mathbb{C}$. First, assume that the polynomial $q$ is even, that is $q(z)=q(-z), z \in \mathbb{C}$. Then, it follows from (50) that $\log (q(z))$ is a rational function so that $q$ can only be a constant, namely 1 by the normalization (31) of the weight $w$. Second, assume that the polynomial $q$ is not even (hence different from a constant). It implies the existence of some root $\alpha \in \mathbb{C}$ of $q$ such that either $q(-\alpha) \neq 0$ or $-\alpha$ is a root of $q$ of different multiplicity than that of $\alpha$. Note that $\alpha \neq 0$ since, by assumption, $q(0)>0$. Then we get a contradiction. Indeed, in view of the definition (44) of $B_{N}$, we readily observe that the left-hand side of (50) has a branch point at $\alpha$ while the right-hand side has not. Hence, $q(z)$ is constant, equal to 1, and the proof of Proposition 2 is finished.

\subsection{Proof of Proposition 3}

Choosing $p=2$ and $g=\left|\log \left(w_{0}\right) w\right|^{1 / 2} \in L^{1}$ in Theorem 2 of [1] shows that

$$
\liminf _{n \rightarrow \infty} \int_{\Delta}\left|\log \left(w_{0}(x)\right)\right| p_{n}^{2}(x) w(x) d x \geq \int_{\Delta}\left|\log \left(w_{0}(x)\right)\right| \rho(x) d x
$$

for any weight $w$ in the Erdös-Turan class $\mathcal{E} \mathcal{T}$. If $\log ^{+}\left(w_{0}\right) \in L^{\infty}$, there exists a constant $C>1$ such that $w_{0}(x) \leq C, x \in \Delta$. Hence $\left|\log \left(w_{0} / C\right)\right|=-\log \left(w_{0} / C\right)$ and substracting $\log (C)$ to both sides of (51), we get (26) since

$$
\int_{\Delta} \rho(x) d x=\int_{\Delta} p_{n}^{2}(x) w(x) d x=1 .
$$

A similar reasonning shows (27) when $\log ^{-}\left(w_{0}\right) \in L^{\infty}$. Since this argument applies for any weight in the Erdős-Turan class, the last assertion in the proposition also follows.

\section{Acknowledgement}

This work was partially supported by INTAS project 2000-272 (B.B., A.M.F. and F.W.), a research grant from the Ministry of Science and Technology (MCYT) of Spain, project code BFM2001-3878-C02 (B.B and A.M.F.), by Junta de Andalucía, Grupo de Investigación FQM 0229 and by the Ministry of Education, Culture and Sports of Spain through the grant PR2003-0104 (A.M.F.). A.M.F. wishes to acknowledge also the hospitality of the Department of Mathematics, Universite de Lille I, France, where this work was started.

E.A.R. acknowledges also the support of a research grant from the Ministry of Science and Technology (MCYT) of Spain, project code SAB2001-0120, and the hospitality of the University of Almería. 


\section{References}

[1] A. I. Aptekarev, V. S. Buyarov, and J. S. Dehesa. Asymptotic behavior of the $L^{p}$-norms and the entropy for general orthogonal polynomials. Russian Acad. Sci. Sb. Math., 82(2):373-395, 1995.

[2] N. K. Bari. A treatise on trigonometric series, Pergamon Press, Oxford, 1964.

[3] I. Bialynicki-Birula and J. Mycielsky. Uncertainty Relations for Information Entropy in Wave Mechanics. Commun. Math. Phys., 44:129-132, 1975.

[4] J. S. Dehesa, W. Van Assche, and R. J. Yáñez. Information entropy of classical orthogonal polynomials and their application to the harmonic oscillator and Coulomb potentials. Methods and Appl. Analysis, 4:91-110, 1997.

[5] J. S. Dehesa, A. Martínez-Finkelshtein, and J. Sánchez-Ruiz. Quantum Information Entropies and Orthogonal Polynomials. J. Comput. Appl. Math., 133:23-46, 2001.

[6] J.S. Dehesa, W. Van Assche, R.J. Yáñez, Information entropy of classical orthogonal polynomials and their application to the harmonic oscillator and Coulomb potentials, Meth. Appl. Anal. 4 (1997) 91-110.

[7] R.M. Dreizler and E.K.U. Gross. Density Functional Theory: An Approach to the Quantum Mechanics. Springer-Verlag, Heidelberg, 1990.

[8] R. Killip and B. Simon. Sum rules for Jacobi matrices and their applications to spectral theory. Annals of Math., 158:253-321, 2003.

[9] A. L. Levin and D. S. Lubinsky. Orthogonal Polynomials with Exponential Weights, volume 4 of CMS Books in Mathematics. Springer Verlag, 2001.

[10] E. Levin and D. S. Lubinsky. Asymptotics for entropy integrals associated with exponential weights. J. Comput. Appl. Math., 156:265-283, 2003.

[11] A. Maté, P. Nevai and V. Totik. Necessary conditions for weighted mean convergence of Fourier series in orthogonal polynomials. J. Approx. Theory, 46:314-322, 1986.

[12] R.G. Parr, W. Yang, Density Functional Theory of Atoms and Molecules, Oxford University Press, New York, 1989.

[13] E. A. Rakhmanov. On the asymptotics of the ratio of orthogonal polynomials. Math. USSR Sb., 32:199-213, 1977.

[14] C.E. Shannon, A mathematical theory of communication, Bell Syst. Tech. J. 27 (1948) 379-423, 623-656; reprinted in: The Mathematical Theory of Communication, Eds. C. E. Shannon and W. Weaver, University of Illinois Press, Urbana, 1949.

[15] R.J. Yáñez, W. Van Assche, J.S. Dehesa, Position and momentum information entropies of the D-dimensional harmonic oscillator and hydrogen atom, Phys. Rev. A 50 (1994) 3065-3079.

[16] R. J. Yañez, W. Van Assche, R. González-Férez, and J. S. Dehesa. Entropic integrals of hyperspherical harmonics and spatial entropy of $D$-dimensional central potentials. J. Math. Physics, 40(11):5675-5686, 1999. 